\title{
Hyperglycemia induces osteogenic differentiation of tendon derived mesenchymal stem cells
}

\author{
Giai Via $\mathrm{A}^{1 *}$, McCarthy MB², Francke $\mathrm{M}^{2}$, Pipino $\mathrm{G}^{3,4}$, Oliva F $\mathrm{F}^{5}$, Mazzocca $\mathrm{AD}^{6}$ and Maffulli $\mathrm{N}^{7,8}$ \\ ${ }^{1}$ Department of Musculoskeletal Disorders, Orthopaedic and Traumatology Surgery, San Camillo Forlanini, Circonvallazione Ganicolense 87, Rome, Italy \\ ${ }^{2}$ Department of Orthopaedic Surgery, Connecticut, USA Human Soft Tissue Research Laboratory, UConn Musculoskeletal Institute, University of Connecticut, \\ 263 Farmington Avenue, Farmington, CT 06030, USA \\ ${ }^{3}$ Department of Orthopaedic Surgery and Traumatology Villa Regina Hospital Bologna, Italy \\ ${ }^{4}$ Department of Orthopedics and Physiotherapy of UCM University, Italy \\ ${ }^{5}$ Department of Musculoskeletal Disorders, School of Medicine and Surgery, University of Salerno, Salerno, Italy \\ ${ }^{6}$ Department of Orthopaedic Surgery, UConn Musculoskeletal Institute, University of Connecticut, 263 Farmington Avenue, Farmington, CT 06030, USA \\ ${ }^{7}$ Department of Musculoskeletal Disorders, School of Medicine and Surgery, University of Salerno, Salerno, Italy \\ ${ }^{8}$ Mary University of London, Barts and the London School of Medicine and Dentistry, Centre for Sports and Exercise Medicine, Mile End Hospital, 275 Bancroft \\ Road, London E1 4DG, England
}

\begin{abstract}
Introduction: The physiopathology of calcific tendinopathy is largely unknown, but it could be the result of an active cell-mediated process. Many endocrine and metabolic diseases may impair the homeostasis of the tendon, and recent studies focus on the influence of extracellular matrix on the differentiation pathway of mesenchymal stem cells (MSCs). The present study investigates whether hyperglycemia may influence the differentiation of tendon derived-MSCs (TD-MSCs) into an osteoblast lineage.

Methods: MSCs were harvested from discarded human tendon excised during arthroscopic rotator cuff repair. The resulting TD-MSCs were counted, plated and grown to confluence in supplemented medium ( $\alpha$ MEM). TD-MSCs were treated with $\alpha$ MEM containing low $(5.0 \mathrm{mM})$, physiological (10 mM) or high $(25 \mathrm{mM})$ glucose, (+) and (-) $10^{-10} \mathrm{mM}$ insulin for 0 , and 24 hours, 7, 14 and 30 days. Control cells were treated with $\alpha M E M$. Only quantitative polymerase chain reaction (qPCR) was used to measure changes in gene expression levels specific for fibrocartilage of TD-MSCs (collagen type I-II-III, aggrecan, osteopontin, fibronectin and alkaline phosphatase). Calcium levels were measured after 30 days in culture. Immunohistochemistry staining was used to determine the amount of the specific proteins present in each group tested.
\end{abstract}

Results: There was an increased gene expression of collagen type I, alkaline phosphatase, aggrecan and osteopontin in TD-MSCs supplemented with high glucose compared to other groups ( $\mathrm{p}<0.05$ ). When insulin was added to the $\alpha \mathrm{MEM}$, a higher increase of collagen type I and III was found in TD-MSCs cultured with high dose glucose. The synthesis of alkaline phosphatase and the expression of aggrecan and osteopontin genes were higher in TD-MSCs cultured with high dose glucose and high dose glucose with insulin. After 30 days, calcium content was increased in the high glucose group and in the high glucose medium and insulin.

Conclusions: When cultured in a high glucose medium, TD-MSCs express bone markers, and are able to differentiate toward an osteoblast lineage. These results reinforce the concept that calcific tendinopathy may be caused by erroneous differentiation of MSCs in the presence of high levels of serum glucose.

\begin{abstract}
Abbreviations: MSCs: Mesenchymal stem cells; TD-MSCs: Tendon-derived mesenchymal stem cells; CT: Calcific tendinopathy; MEM: Minimal essential media; LG: Low glucose; PH: Physiological glucose; HG: High glucose; LG-I: Low glucose (+) insulin; PHG-I: Physiological glucose (+) insulin; HG-I: High glucose (+) insulin.

\section{Introduction}

Calcific tendinopathy (CT) is characterized by calcium carbonate apatite deposits in the extracellular matrix (ECM) of the tendons. The physiopathology of CT is still controversial and largely unknown, especially because it is difficult to clarify the first steps of the pathogenetic process. However, rather than caused by a simple precipitation of calcium, CT seems to be the result of an active cellmediated process [1]. Recently some authors suggested that the ectopic bone formation into the tendon may be caused by the erroneous
\end{abstract}

differentiation of resident mesenchymal stem cells (MSCs) [2-4]. However, the mechanism that causes this erroneous differentiation is still not elucidated. This process can involve the tendon-bone junction (insertional CT), or the midsubstance of the tendons. Insertional CT has a tridimensional structure similar to the trabecular bone [5], and typically involves the Achilles or the patellar tendon. Calcific deposits

${ }^{\star}$ Correspondence to: Alessio Giai Via, MD, Department of Musculoskeletal Disorders, Orthopaedic and Traumatology Surgery, San Camillo Forlanini, Circonvallazione Ganicolense 87, Rome, Italy, E-mail: agiaivia@unisa.it

Key words: calcific tendinopathy, tendons, calcific deposits, mesenchymal stem cells, hyperglycemia, diabetes mellitus

Received: August 12, 2020; Accepted: August 28, 2020; Published: August 31, 2020 
of the rotator cuff involves the midportion of the tendon, and they have different clinical and histological features, more similar to a toothpastelike material [1]

The main hypothesis on the pathogenesis of tendinopathy is a failed healing response [6]. Accordingly, intrinsic and extrinsic factors impair the homeostasis of tendons, and, if at any stage the healing process is impaired or disrupted, the normal healing may be diverted into an abnormal pathway. There is increasing evidence in the literature about the influence of hormones and metabolic diseases, such hypothyroidism, obesity and diabetes mellitus, on tendons homeostasis and self-healing capability [7-9]. Some authors reported worse results after rotator cuff repair and an higher incidence of CT in diabetic patients [10]. More than $30 \%$ of patients with insulin-dependent diabetes have tendon calcifications. Recently, poorer results have been reported in patients with diabetes mellitus after open arthrolysis for elbow stiffness, compared to patients with normal glycemia [11]. Snedeker et al. have recently elucidated the relationship between diabetes mellitus and the changes of the tendon ECM, showing that the crosslink between advanced glycation end-products (AGEs) and collagen, ECM proteins and tenocytes, modifies the mechanical properties of connective tissues and the healing capacity of tendons [12].

The present study investigated whether hyperglycemia may influence the differentiation of tendon derived-MSCs (TD-MSCs) into an osteoblast lineage. We hypothesized that high glucose levels negatively affect the expression of genes specific for fibrocartilage of adult human TD-MSCs.

\section{Materials and methods}

Bone marrow aspiration and TD-MSC characterization: TDMSCs were harvested from discarded human tendon excised during arthroscopic rotator cuff repair (Institutional Review Board \# IE-06577-2). Tissue was immediately taken to the laboratory and digested overnight with type I collagenase $(2.0 \mathrm{mg} / \mathrm{ml})$ in minimal essential media (MEM). The resulting TD-MSCs were counted, plated and grown to confluence in supplemented complete MEM (aMEM), containing $10 \%$ fetal bovine serum (FBS) and $0.1 \%$ penicillin/streptomycin. Prior to experimentation, TD-MSCs were grown for 24 hours in glucose free aMEM. After 24 hours, cells were treated with aMEM containing low $(5 \mathrm{mM})$, physiological $(10 \mathrm{mM})$ and high $(25 \mathrm{mM})$ glucose, $(+)$ and (-) $10^{-10} \mathrm{mM}$ insulin for 0 , and 24 hours and 7,14 and 30 days. Control cells were treated with complete aMEM alone. Quantitative polymerase chain reaction (qPCR) was used to measure changes in gene expression levels specific for fibrocartilage of TD-MSCs (Collagens type I, II, and III, Alkaline phosphatase, Aggrecan and Osteopontin). Calcium levels were measured after 30 days in culture. Immunohistochemistry staining was used to determine the amount of the specific proteins present in each group tested.

Fluorescence-activated cell sorting (FACS) analysis: FACS analysis was used to confirm a positive phenotype for stem cells digested from extracted rotator cuff tendon. Once confluence was achieved, cells were typsinized in $0.25 \%$ trypsin/EDTA. The pellet was resuspended in staining buffer containing $1 \%$ bovine serum albumin, 1\% human serum, and $1 \%$ fetal bovine serum in PBS buffer. Cells were incubated with either fluorescein isothiocyanate (FITC) or phycoerythrin (PE) antibodies, washed with staining buffer, and analyzed using a FACSCalibur (BD Biosciences, San Jose, California). For positive analysis of stem cells, phycoerythrin-conjugated mouse monoclonal anti-CD73 immunoglobulin G (IgG), anti-CD90 IgG, anti-CD105 IgG were used (BD Biosciences). For the negative control, fluorescein isothiocyanate-conjugated anti-mouse CD45 monoclonal IgG (BD Biosciences) was used. All antibodies were reactive against human antigens.

Experimental design: TD-MSCs were plated onto well Primaria tissue culture plates (Fisher Scientific, Agawam, MA) for 24 hours at a concentration of 40,00 cells $/ \mathrm{cm} 2$ containing glucose free aMEM. Only passage 1 cells were used [13]. After 24 hours, cells were treated with complete MEM supplemented with low $(5 \mathrm{mM})$, physiological $(10 \mathrm{mM})$ and high $(25 \mathrm{mM})$ glucose (Sigma, St Louis, MO) for 0, and $24 \mathrm{hrs.}$, 7 and 14 days. $10^{-10} \mathrm{mM}$ insulin (Sigma) were added (+) or not (-) to the medium. The calcium content was measured after 4 weeks. Control cells were treated with complete aMEM alone. Media for all wells was changed every 3 days. All experiments were performed in triplicate using TD-MSCs obtained from 3 different patients.

\section{Quantitative polymerase chain reaction (qPCR)}

Estabilished gene markers were chosen to quantify a change in the ability of TD-MSCs to express genes associated with calcific tendinopathy (type I, II and III collagen, alkaline phosphatase, osteopontin and aggrecan). Cells were first grown in culture for 24 hours in glucose free medium followed by 0, 24 hours, 7 and 14 days in low, physiological and high concentration of glucose, with (+) or without (-) the addition of $10^{-10} \mathrm{mM}$ of insulin, as described previously. RNA was isolated from cells using the TRIzol reagent (Thermo Fisher Scientific). Quantity and purity of the RNA were measured using a NanoDrop (Thermo Fisher Scientific). RNA was reverse transcribed into cDNA using $1 \mu \mathrm{g}$ messenger RNA and a High Capacity Reverse Transcription kit (Thermo Fisher Scientific). The qPCR was performed using $200 \mathrm{ng}$ of cDNA as a template, in triplicate, using the StepOne Real-Time PCR System (Applied BioSystems, Foster City, California). For all samples, Glyceradehyde 3-phosphate dehydrogenase (GAPDH) was used as the endogenous control [14]. Three qPCR assays were performed for each of 3-patients with 3 replicates per each experimental condition.

Calcium and DNA content: Calcium was extracted twice for 30 min. each from the cells using 5\% Trichloroacetic Acid (TCA) after 4 weeks in culture. Using a calcium kit (Sigma, St Louis, MO) calcium content was measured in the cell extract. To normalize DNA to mineralization, cells were disrupted by adding $0.01 \%$ SDS to each well for $15 \mathrm{~min}$. For each sample, $100 \mu \mathrm{l}$ of filtered Hoechst dye $(10 \mu \mathrm{g} / \mathrm{ml})$ was added to a well in a clean 96 well plate with $100 \mu$ lof cell lysate. 30 $\mathrm{nm}$ excitation and $460 \mathrm{~nm}$ emission using a microplate reader (BioTEK Synergy HT, Winooski, VT) was used to measure DNA content. Three assays were performed for each of 3 patients with six-replicates per each experimental condition.

\section{Statistics}

Data are expressed as the mean \pm standard error or as $95 \%$ confidence intervals of the technical triplicates of 6-9 independent samples. Descriptive statistics are reported as mean and standard deviation. The Kruskal-Wallis test was used for all comparisons. When significant, the Wilcoxon rank-sum test was used for pairwise comparisons of calcium content, and the Dunn's test for comparisons of gene expression data. The alpha level for all statistics was set at 0.05 . All analysis was performed with Stata 12 (StataCorp. 2011. Stata Statistical Software: Release 12. College Station, TX: StataCorp LP.).

\section{Results}

TD-MSCs characterization by colony forming unit assay and FACS analysis: A CFU assay was performed to determine whether the 
digested cells had properties indicative of stem cells such as the ability to adhere to tissue culture plastic and form colonies. After 2-3 days in culture, cells had the ability to adhere to tissue culture plastic and were able to give rise to variable-sized colonies after 7-10 days in culture. Colony forming units (CFU's) were counted after 7-10 days in culture to evaluate the number of TD-MSCs; $24.80+18.6$ CFU's grew/106 nucleated cells resulting in $1430+1590 \mathrm{TD}-\mathrm{MSCs} / \mathrm{ml}$. TD-MSCs also expressed surface markers to be found on adult human stem cells: CD73 (90.50\% + 0.1\%), CD90 (96.25\% + 0.50\%), and CD105 $(98.90 \%$ $+0.50 \%)$, but were negative for hematopoietic surface marker CD45 $(0.15 \%+0.15 \%)$.

\section{Gene expression}

Type I and type II Collagen genes expression were significantly increased at 7 days in the high glucose group compared to all other groups within their respective time points $(\mathrm{p}=0.02$ and 0.00063 respectively). At 14 days, the highest expression of Collagen type II was seen in the high glucose MEM, while when insulin was added to the MEM in the high glucose group, a significant increase in type I collagen expression was observed at the 14-day time point compared to all other groups at all other times $\mathrm{p}=0.015$. Furthermore, at 14 days type II collagen gene expression was down regulated in the HG-I group compared to 7 days (Figure 1).

After 7 and 14 days in culture, type III collagen gene expression was significantly increased in TD-MSCs supplemented with HG and HG-I compared to control, low and physiological groups ( $\mathrm{p}<0.05)$. The addition of insulin into the MEM increased the synthesis of Type III collagen also in the MSCs cultures with PHG $(\mathrm{p}=0.045)$ (Figure 2).

The aggrecan expression was significantly higher in the HGMEM at 7 days compared to others physiological and low glucose groups (Figure 3$)(p=0.0039)$. When insulin was added to the aMEM, osteopontin gene expression was significantly higher in the HG-I MEM at 14 days compared to physiological and low glucose and insulin culture $(\mathrm{p}=0.05)$.

There was a significantly higher alkaline phosphatase gene expression after 7 and 14 days of culture in the HG-MEM group compared to control, low and physiological glucose groups $(\mathrm{p}=.00081$ and $\mathrm{p}=0.021$ ). At the 14-day time point significantly higher gene expression was seen in the HG-I MEM $(+)$ insulin compared to all other groups at all time points $(\mathrm{p}=0.0050)$ (Figure 4$)$.

Type I Collagen Gene Expression

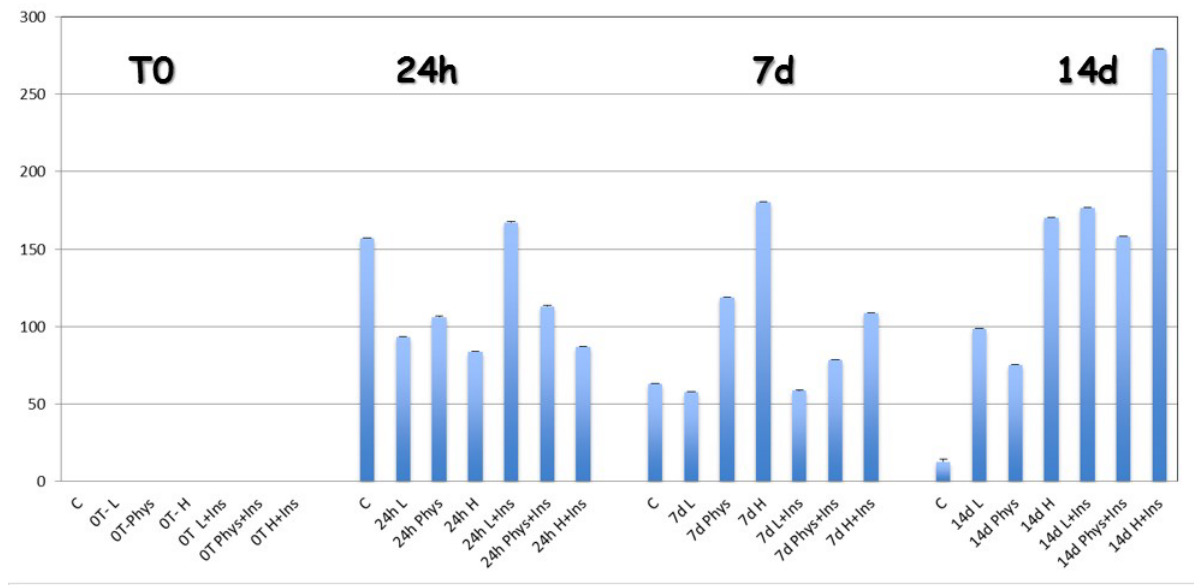

Type II Collagen Gene Expression

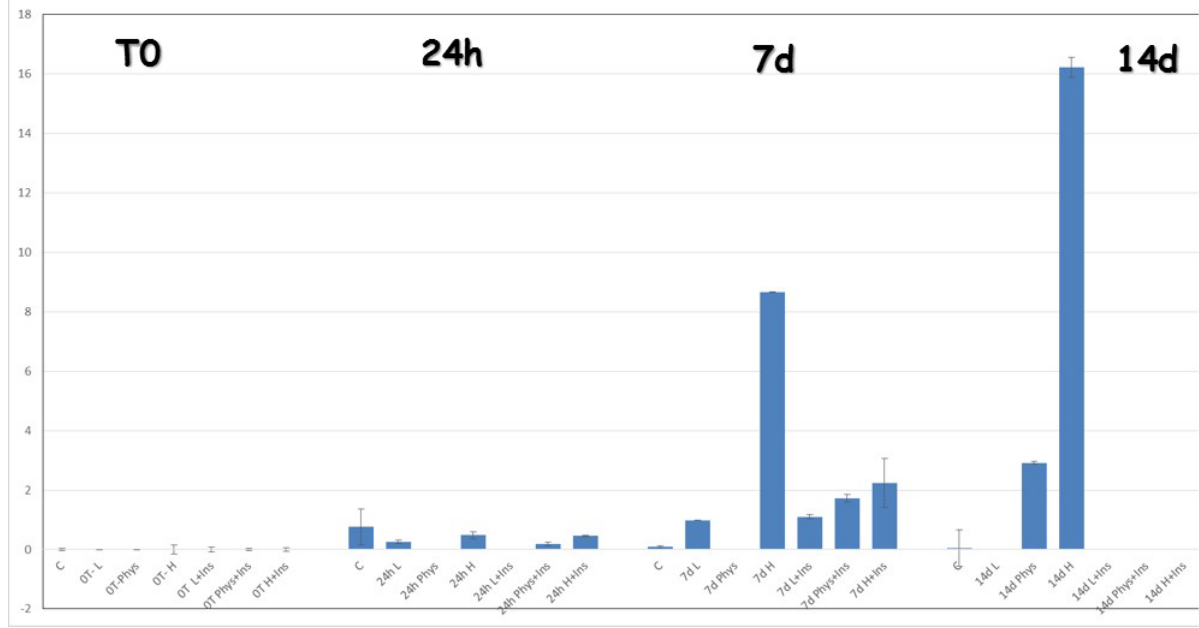

Figure 1. There was a significant increase in type I and type II collagen expression at the 7 and 14 days time point in the HG-group compared to all other times and groups. When insulin was added, the type I collagen expression was significantly increased at 14 days compared to non-insulin treated cells. The highest expression was observed in the HG-I group 
Type III Collagen Gene Expression

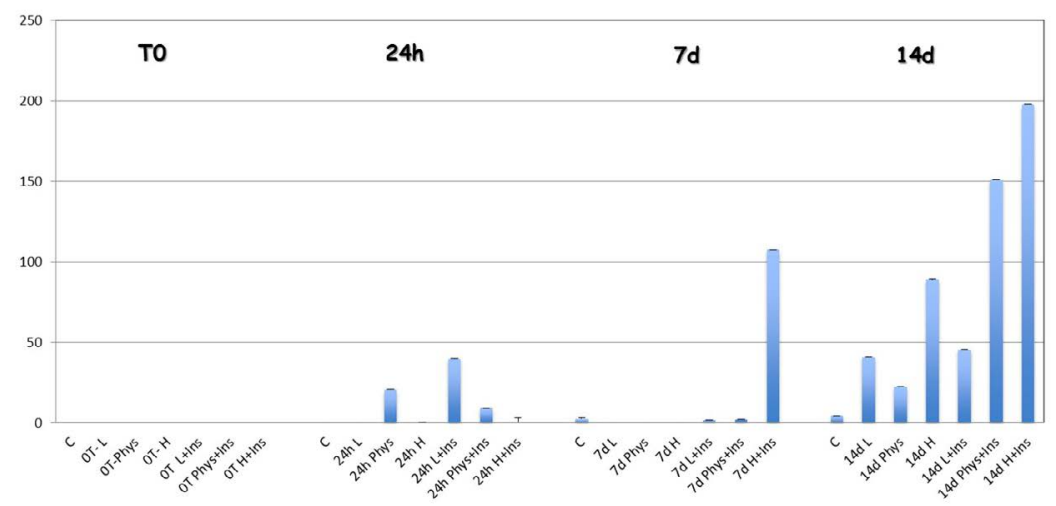

Figure 2. Type III collagen gene expression was significantly increased in TD-MSCs supplemented with HG at 7 days compared to other groups. When TD-MSCs were cultured with the addition of insulin, the gene expression of collagen type III was significantly higher not only in the HG-I cells at 7 and 14 days compared to the other groups, but also in the PHG at 14 compared to non-insulin (-) and LG+insulin MEM
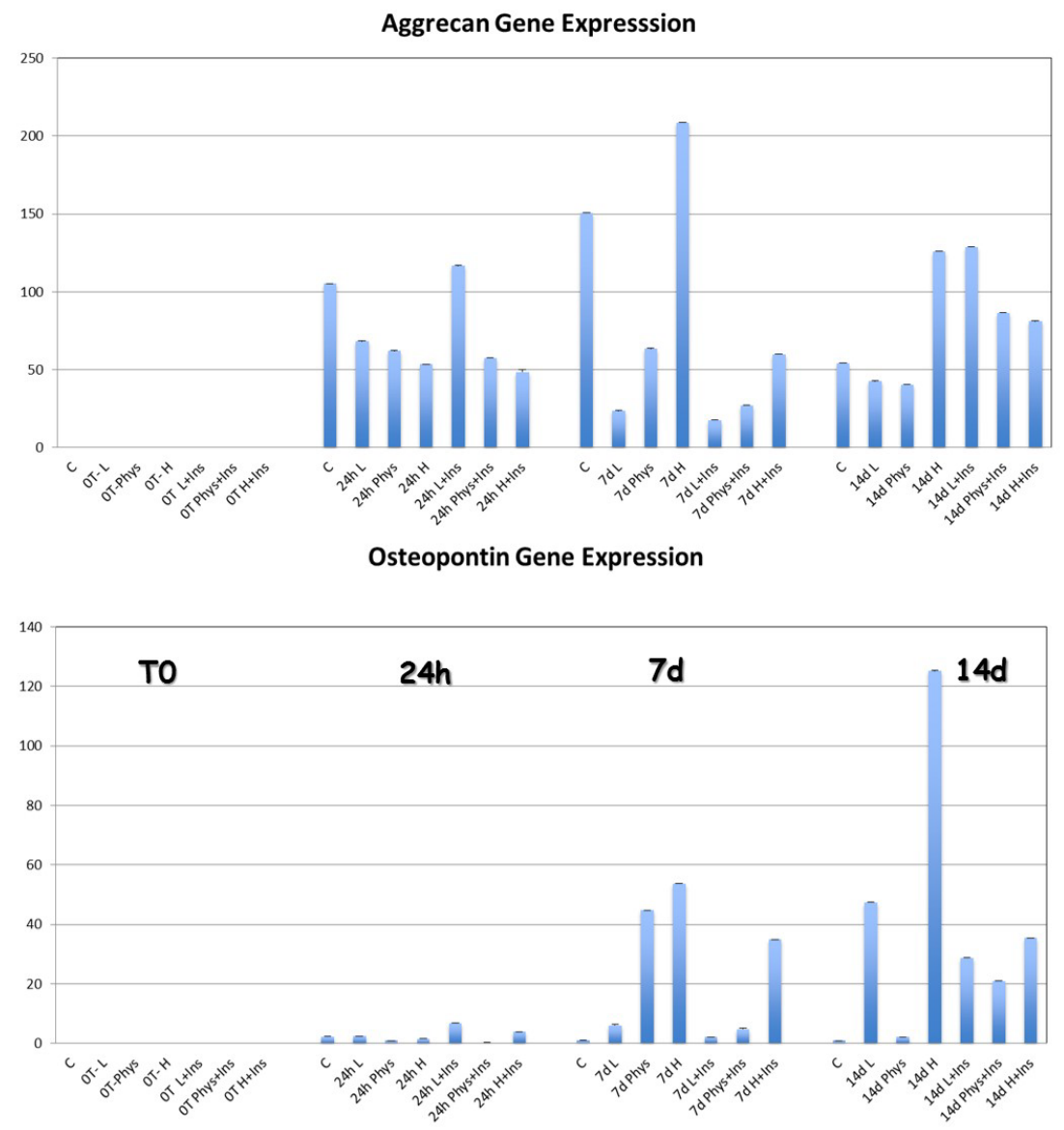

Figure 3. The gene expression for aggrecan was significantly increased in the HG-group at 7 and 14 day compared to LG, PG, and control cells. The osteopontin expression was also higher in the HG-MEM at 7 and 14 days compared to the other groups. When TD-MSCs have been cultured and the addition of insulin, the synthesis of Aggrecan was significantly higher in the LG-I and PHG-I MSCs compared to LG and PHG MSCs at 14 days

\section{Calcium content}

To confirm cellular mineralization, a calcium content assay was performed at 30 days. Calcium was measured in TD-MSCs cultures treated with low $(5 \mathrm{mM})$, physiological $(10 \mathrm{mM})$ and high $(25 \mathrm{mM})$ glucose $(+)$ and (-) insulin. HG with or without insulin significantly stimulated the amount of calcium per $\mu \mathrm{g}$ DNA in TD-MSCs cultures compared to control, LG and PG groups $(+)$ and $(-)$ insulin $(\mathrm{p}<0.05)$. No significant differences were found between the other groups (Figure 4 ).

\section{Microscopic analysis}

At the immunocytochemistry light microscopic analysis, TD-MSCs cultured with HG showed an increase in cytoplasmic staining for type I, 
II and III collagen, aggrecan and osteopontin compared to low glucose and control untreated cells (Figure 5).

\section{Discussion}

The aetiopathogenesis of CT is still largely unknown. Animal studies on the Achilles tendon showed that CT is produced by a process similar to endochondral ossification, with bone formation and remodeling mediated by osteoblasts and osteoclasts, promoting the idea that it could be the result of an active cell-mediated process [15]. In this process, resident MSCs may play an important role. According to these findings, some authors proposed that the erroneous differentiation of MSCs into chondrocytes or osteoblasts could be responsible for insertional CT of

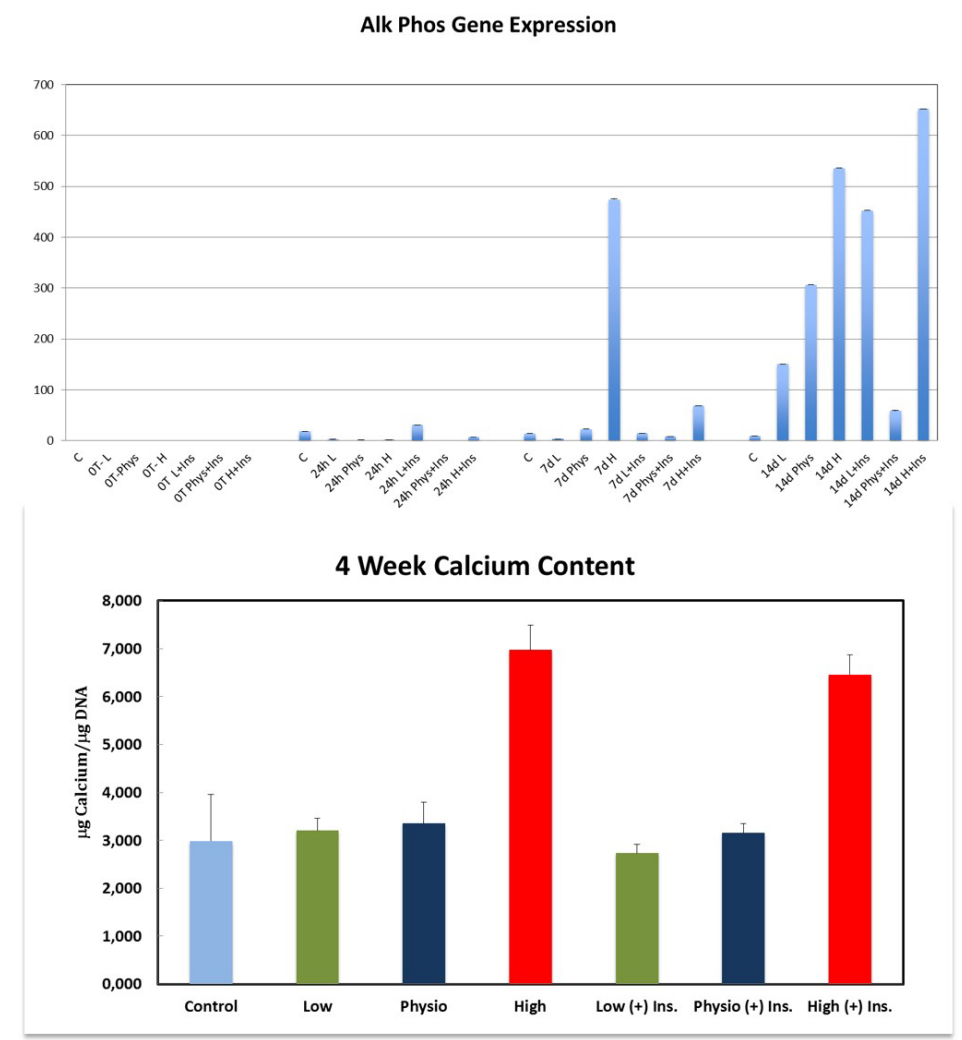

Figure 4. Alkaline phosphatase gene expression was significantly increase at 7 and 14 days in the HG-groups compared to LG, PG and control cells. The highest expression of alkaline phosphatase gene was observed in the HG-I MEM at 14 days compared to all other groups at all time points. This figure shows also the effect of different concentrations of glucose $(+)$ or (-) insulin on mineralization after 4 weeks. The amount of calcium per $\mu \mathrm{g}$ DNA was significantly increased in the HG and HG-I MEMs

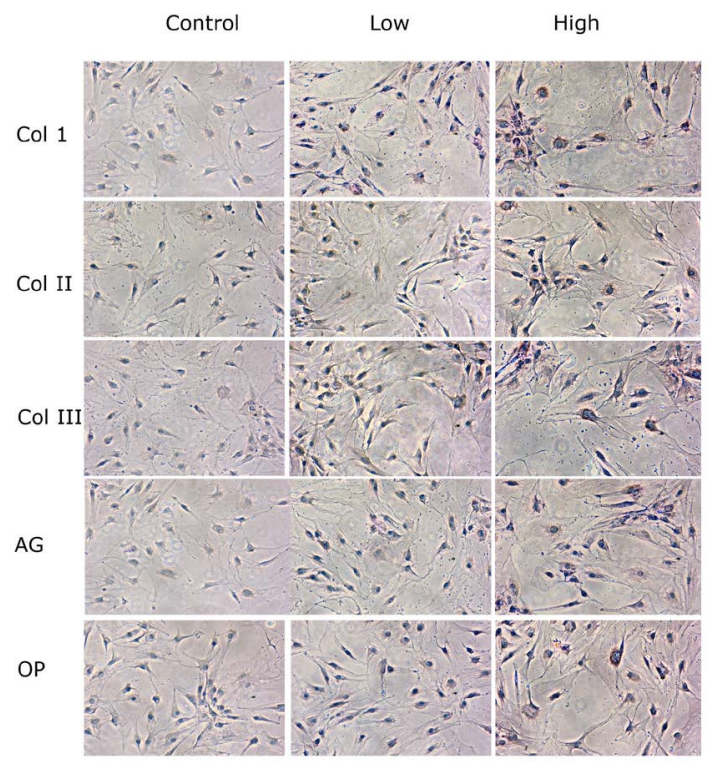

Figure 5. Immunocytochemistry light microscopic analysis of TD-MSCs treated with low (5 mM) and high concentrations ( $25 \mathrm{mM})$ of glucose compared to control cells with no treatment. Proteins deposits are expressed on the surface of the cells (brown color). TD-MSCs treated with high glucose showed an increase in cytoplasmic staining for Type I, II and III Collagen (Coll, II and III), Aggrecan (AG) and Osteopontin (OP) in comparison to low glucose and control untreated cells 
the Achilles tendon [3]. Harada et al. investigated the role of MSCs in the ossification process of the posterior longitudinal ligament of the spine [16], evidencing an increased osteogenic differentiation potential of MSCs: this may be the pathogenetic factor of the calcified ligament.

MSCs have been isolated from various tissues [17], and several studies discussed the adverse effects of MSCs in pathologial conditions, including vascular calcification [18], aortic valve calcification [19], fibrodysplasia ossificans progressive [20], and ectopic ossification following burn injury [ 21]. However, the mechanism leading to the erroneous differentiation of MSCs is still unknown. In this scenario, the microenvironment, which is the substrate where cells live, proliferate and differentiate, plays a key role. It consists of cells, soluble factors, and ECM. MSCs and ECM are in close connection to each other [22]. MSCs are influenced by their microenvironment and respond to a variety of signals which mostly originate from the ECM [23]. These signals are able to regulate stem cell behaviors, proliferation, self-renewal and differentiation [24]. On the other hand, the characteristics of the ECM can be modified by growth factors, proteases, and cytokines produced by MSCs $[25,26]$. Therefore, local modifications of the ECM can affect the differentiation potential of resident MSCs. In vitro, Hagmann et al. [27] showed that the expansion media was able to influence the growth characteristics and differentiation potential of bone marrow MSCs, and their ability to form cartilaginous tissue. Again in vitro, artificial changes to the ECM affect the differentiation program of endothelial MSCs, identifying ECM as a critical regulator of vascular cell differentiation [26]. These authors concluded that ECM is a critical regulator of MSCs differentiation.

The modification of EMC is the key point of the pathogenesis of tendinopathy [28]. But why MSCs should differentiate into an osteoblast lineage in CT? This is still an unsolved question. Liu et al. [29] investigated the effect of high glucose levels on human aortic smooth muscle cells in vitro, by studying the expressions of BMP-2/Cbfa- 1 and alkaline phosphatase (ALP), and the intracellular calcium content. High glucose levels increase the expressions of BMP-2 and Cbfa-1, ALP activity, and intracellular calcium deposition, and high glucose levels can induce the calcification of vascular smooth muscle cells by inducing osteoblastic differentiation and intracellular calcium deposition [29]. The relationship between the extent of ossification and diabetes mellitus in patients with ossification of the posterior longitudinal ligament (OPLL) was examined by Akune et al. [30] Insulin secretory response (insulinogenic index) was significantly associated with the progression of OPLL [30]. Insulin is able to induce many growth and metabolic responses, and plays important roles in the anabolic regulation of bone metabolism [31,32]. It binds not only the insulin receptor substrate-1 (IRS-1), but also insulin-like growth factor-I (IGF-I) receptor, which is an anabolic factor for bone formation [33]. Some studies showed the relationship between insulin and heterotopic calcifications, such as OPLL and atherosclerosis. Bellows et al. [34] conduced an in vitro study on fetal rat bone cell populations. Insulin was able to stimulate osteoprogenitor differentiation, and to induce a dose-dependent increase in the number and size of bone and osteoid nodules. However, the limitation and difference of that study compared to the present study is that the differentiation of the progenitors cells was evaluated by counting the number of bone or osteoid nodules, and the effects on proliferation by measuring their size by image analysis. In vitro, insulin accelerates the calcification in human vascular smooth muscle cells, and stimulates the proliferation and osteogenic differentiation induced by BMP- 2 of rat spinal ligament cells in a time- and dose-dependent manner [35-37].
Type I collagen is the most abundant type of collagen in the human body Lui et al. [38] showed that an increase in type I collagen may account for poor matrix organization in CT. Type II collagen is an important component of the ECM at the tendon-bone junction [39], and the expression of Type II collagen is upregulated in human rotator cuff tendinopathy [40]. By support to our hypothesis, we found a statistically significant increased expression of Collagen Type I and II in TD-MSCs cultured in a high glucose medium. The mRNA and protein expression of aggrecan, and its relationship with ectopic ossification has been observed in a CT model [40]. Osteopontin, a protein produced by osteoblasts, is involved in the anchoring of osteoclasts to the mineral of bone matrix, it has an important role in matrix remodeling, and it is upregulated during chondrocyte terminal differentiation [41]. Therefore, it could play an important role during the formation of calcific deposits. The expression of osteopontin has been investigated in an immunohistological study on rotator cuff CT. The authors found an increased expression of Osteopontin in the calcific area of the supraspinatus tendons compared to healthy tendon [42]. Fibronectin is a high-molecular weight extracellular glycoproteins present in skeletal ECM, which regulates cell adhesion, differentiation and functions. Changes in the expression of fibronectin have been reported during cartilage and bone formation, and fibronectin is extremely important for cells survival during osteoblasts maturation $[43,44]$. We found a higher genes expression of aggrecan, osteopontin and fibronectin in TD-MSCs when cultured with high glucose $(25 \mathrm{mM})$ compared to low and physiological glucose concentrations. Alkaline phosphatase is produced during mineralization in the precalcific stage of calcific tendinopathy, where fibro-cartilaginous metaplasia occurs and tendon fibroblasts transform into chondrocytes [45]. An increased expression of alkaline phosphatase gene has been also found in the present study.

Furthermore, when insulin was added to the medium a significantly higher expression of bone markers was found compared to cells cultured without insulin. These data may support our hypothesis that CT may be caused by the erroneous differentiation of resident TD-MSCs in the presence of high glucose levels. Furthermore, a higher expression of bone markers was found in MSCs cultured with high glucose levels and insulin, implying that insulin may act in synergy with glucose and stimulate the osteogenic differentiation of MSCs. To confirm this hypothesis, we recently showed that, when cultured in a high glucose medium, bone marrow-MSCs express bone markers and are able to differentiate into osteoblasts [46]. This could explain the high incidence of insertional CT in patients with diabetes mellitus. As bone marrowMSCs are precommetted, we repeated the experiment with TD-MSCs.

Our experiments have some limitations. The differentiation properties of MSCs may be influenced by donor variation (age, sex, comorbidities of the patient), the culture media, and the passage proliferation. Even though the donor sample was not uniform, the cells were isolated and expanded in the same way, suggesting that the culture conditions (high dose glucose and the addition of insulin) were a significant variable on MSCs differentiation. Then, we used TD-MSCs harvested from the rotator cuff tendons for our experiment. We are conscious that the histological feature of RC calcifications is different from insertional CT, which are more similar to mature bone, and our experiment does not solve the question why these two kinds of calcifications look so different. This allowed us to speculate on the pathogenesis of insertional CT, but we are not sure whether tendonderived MSCs act in the same way. We can only speculate that the different $\mathrm{PH}$ condition between the ECM of the tendon and tendonbone insertion may influence the complete maturation of osteoblast and bone formation. 
In summary, the pathogenesis of tendinopathy is multifactorial, but the precise role of each predisposing factor is not completely understood. The main hypothesis, which tries to explain the pathogenesis of tendinopathy, is the failed healing response [6]. According to this, many intrinsic and extrinsic factors impair the homeostasis of tendons, and, if disturbances at any stages of the healing process occur, the normal healing may be diverted into an abnormal pathway. Recent evidence shows that metabolic disorders and endocrine pathologies can be involved in the etiopathogenesis of tendinopathy [47-49]. In particular, diabetes mellitus is able to modify the ECM of tendons by proteins crosslinking [50]. We believe that the modifications of the microenvironment of the tendon ECM in the course of tendinopathy, together with high glucose and insulin levels, may influence the differentiation of resident MSCs, which is diverted toward the osteoblast lineage.

\section{Conclusion}

TD-MSCs express bone markers if cultured in a high glucose medium, and they are able to differentiate toward an osteoblast lineage. The addition of insulin enhances the differentiation of TD-MSCs. Metabolic diseases may affect the homeostasis of tendons, and CT may be caused by erroneous differentiation of MSCs. Better understanding of the pathogenesis of $\mathrm{CT}$ is essential for development of effective treatment strategies and for the improvement of clinical outcomes.

\section{Author's contributions}

Giai Via designed the study, reviewd the literature and wrote the paper. M.B. McCarthy and M. Francke performed the laboratory study, characterized TD-MSCs and performed the experiment in the laboratory of UConn University. G. Pipino and F. Oliva reviewed the literature and review the present manuscript, Prof. A.D. Mazzocca performed the shoulder arthroscopies and supervised the study, Prof. $\mathrm{N}$. Maffulli supervised the study and reviewed the manuscript.

\section{Competing interests}

A. Giai Via, M. Francke, G. Pipino, F. Oliva, and N. Maffulli declare no competing interests. M.B. McCurthy: received intellectual property royalties from Arthrex. A.D. Mazzocca is a consultant for Orthofix and Arthrex and receives research grants from Arthrex. These companies had no influence on study design and results.

\section{Ethics approval and consent to participate}

All patients sign an informed consent for shoulder arthroscopy and to participate to the study. The study was approved by the ethics committee of University of Connecticut, Institutional Review Board \# IE-06-577-2.

\section{Availability of data and materials}

All data generated or analysed during this study are included in this published article [and its supplementary information files]. The datasets used and/or analyzed during the current study are also available from the corresponding author on reasonable request.

\section{Conflicts of interest}

The authors declare no financial support or other benefits from commercial sources for the work reported on in the manuscript, nor any other financial interests which could create a potential conflict of interest or the appearance of a conflict of interest with regard to the work.

\section{References}

1. Oliva F, Via AG, Maffulli N (2012) Physiopathology of intratendinous calcific deposition. BMC Med 10: 95 .

2. Rui YF, Lui PP, Ni M, Chan LS, Lee YW, et al. (2010) Mechanical loading increased BMP-2 expression which promoted osteogenic differentiation of tendon-derived stem cells. J Orthop Res 29: 390-396. [Crossref]

3. Rui YF, Lui PP, Chan LS, Chan KM, Fu SC, et al. (2011) Does erroneous differentiation of tendon-derived stem cells contribute to the pathogenesis of calcifying tendinopathy? Chin Med J 124: 606-610.

4. Zhang J, Wang JH (2012) BMP-2 mediates PGE(2)-induced reduction of proliferation and osteogenic differentiation of human tendon stem cells. J Orthop Res 30: 47-52. [Crossref]

5. Benjamin M, Rufai A, Ralphs JR (2000) The mechanism of formation of bonny spurs (enthesophytes) in the Achilles tendon. Arthritis \& Rheumatism 43: 576-583.

6. Sharma P, Maffulli N (2005) Tendon injury and tendinopathy: Healing and repair. $J$ Bone Joint Surg Am 87: 187-202

7. Oliva F, Piccirilli E, Berardi AC, Tarantino U, Maffulli N, et al. (2016) Influence of thyroid hormones on tendon homeostasis. Adv Exp Med Biol 920: 133-138

8. Oliva F, Piccirilli E, Berardi AC, Frizziero A, Tarantino U, et al. (2016) Hormones and tendinopathies: the current evidence. Br Med Bull 117: 39-58

9. Zreik NH, Malik RA, Charalambous CP (2016) Adhesive capsulitis of the shoulder and diabetes: a meta-analysis of prevalence. Muscles Ligaments Tendons J 6: 26-34.

10. Oliva F, Via AG, Maffulli N (2011) Calcific tendinopathy of the rotator cuff tendons. Sports Med Arthrosc 19: 237-243. [Crossref]

11. Sun Z, Xiong H, Fan C (2018) Impact of different glucose metabolism status on clinica outcomes of open arthrolysis for posttraumatic elbow stiffness. J Shoulder Elbow Surg 27: 1072-1077.

12. Snedeker JG, Gautieri A. The role of collagen crosslinks in ageing and diabetes - the good, the bad, and the ugly. Muscles Ligaments Tendons J 17: 303-308

13. Yao L, Bestwick CS, Bestwick LA, Maffulli N, Aspden RM, et al. (2006) Phenotypic drift in human tenocyte culture. Tissue Eng 12: 1843-1849.

14. Globus RK, Doty SB, Lull JC, Holmuhamedov E, Humphries MJ, et al. (1998) Fibronectin is a survival factor for differentiated osteoblasts. J Cell Sci 111: 1385-1393

15. Lui PP, Fu SC, Chan LS, Hung LK, Chan KM, et al. (2009) Chondrocyte phenotype and ectopic ossification in collagenase-induced tendon degeneration. $J$ Histochem Cytochem 57: 91-100. [Crossref]

16. Harada Y, Furukawa K, Asari T (2014) Osteogenic lineage commitment of mesenchymal stem cells from patients with ossification of the posterior longitudinal ligament. Biochem Biophys Res Commun 17: 1014-1020

17. Giai Via A, Frizziero A, Oliva F (2012) Biological properties of mesenchymal stem cells from different sources. Muscles Ligaments Tendons $J$ 16: 154-162

18. Tang R, Gao M, Wu M, Liu H, Zhang X, et al. (2012) High glucose mediates endothelial-to-chondrocyte transition in human aortic endothelial cells. Cardiovasc Diabetol 22: 113 .

19. Chen JH, Yip CY, Sone ED (2009) Identification and characterization of aortic valve mesenchymal progenitor cells with robust osteogenic calcification potential. Am J Pathol 174: 1109-1119. [Crossref]

20. Medici D, Shore EM, Lounev VY (2010) Conversion of vascular endothelial cells into multipotent stem-like cells. Nat. Med 16: 1400-1406

21. Nelson ER, Wong VW, Krebsbach PH (2012) Heterotopic ossification following burn injury: the role of stem cells. J Burn Care Res 33: 463-470

22. Da Silva SV, Renovato-Martins M, Ribeiro-Pereira C, Citelli M, Barja-Fidalgo C, et al. (2016) Obesity modifies bone marrow microenvironment and directs bone marrow mesenchymal cells to adipogenesis. Obesity.

23. Mardones R, Jofré CM, Minguell JJ (2015) Cell Therapy and tissue engineering approaches for cartilage repair and/or regeneration. Int J Stem Cells 8: 10-13. [Crossref]

24. Yang G, Rothrauff BB, Lin H, Yu S, Tuan R, et al. (2016) Tendon-derived extracellular matrix enhances TGF- $\beta 3$ induced tenogenic differentiation of human adipose-derived stem cells. Tissue Eng Part A.

25. Mardones R, Giai Via A, Jofré C, Minguell J, Rodriguez C, et al. (2016) Cell therapy for cartilage defects of the hip. Muscles, Ligaments and Tendons $J$ 6: 361-366 
26. Lozito TP, Taboas JM, Kuo CK, Tuan RS (2009) Mesenchymal stem cell modification of endothelial matrix regulates their vascular differentiation. $J$ Cell Biochem 107: 706713

27. Hagmann S, Moradi B, Frank S, Dreher T, Kämmerer PW, et al. (2013) Different culture media affect growth characteristics, surface marker distribution and chondrogenic differentiation of human bone marrow-derived mesenchymal stromal cells. $B M C$ Musculoskelet Disord 30: 223

28. Giai Via A, Papa G, Oliva F, Maffulli N (2016) Tendinopathy. Curr Phys Med Rehabil Rep 2016.

29. Liu F, Zhong H, Liang JY (2010) Effect of high glucose levels on the calcification of vascular smooth muscle cells by inducing osteoblastic differentiation and intracellular calcium deposition via BMP-2/Cbfo-1 pathway. J Zhejiang Univ Sci B 11: 905-911

30. Akune T, Ogata N, Seichi Ai, Ohnishi I, Nakamura K, et al. (2001) insulin secretory response is positively associated with the extent of ossification of the posterior longitudinal ligament of the spine. JBJS 83: 1537-1544. [Crossref]

31. Thomas DM, Hards DK, Rogers SD, Ng KW, Best JD, et al. (1996) Insulin receptor expression in bone. J Bone Miner Res 11: 1312-1320

32. Mazzocca AD, McCarthy MB, Chowaniec D, Cote MP, Judson CH, et al. (2011) Bone marrow-derived mesenchymal stem cells obtained during arthroscopic rotator cuff repair surgery show potential for tendon cell differentiation after treatment with insulin. Arthroscopy 27: 1459-1471.

33. Canalis $\mathrm{E}$ (1993) Insulin like growth factors and the local regulation of bone formation. Bone 14: 273-276.

34. Bellows CG, Jia D, Jia Y (2006) Different effects of insulin and insulin-like growth factors I and II on osteoprogenitors and adipocyte progenitors in fetal rat bone cell populations. Calcif Tissue Int 79: 57-65

35. Yano H, Ohya K, Amagasa T (1994) Effects of insulin on in vitro bone formation in fetal rat parietal bone. Endocr J 41: 293-300

36. Olesen P, Nguyen K, Wogensen L (2007) Calcification of human vascular smooth muscle cells: associations with osteoprotegerin expression and acceleration by highdose insulin. Am J Physiol Heart Circ Physiol 292: 1058-1064.

37. Li H, Liu D, Zhao CQ, Jiang LS, Dai LY, et al. (2008) Insulin potentiates the proliferation and bone morphogenetic protein-2-induced osteogenic differentiation of rat spinal ligament cells via extracellular signal-regulated kinase and phosphatidylinositol 3-kinase. Spine 33: 2394-2402.
38. Lui PP, Chan LS, Lee W, Fu SC, Chan KM, et al. (2010) Sustained expression of proteoglycans and collagen type III/type I ratio in a calcified tendinopathy model. Rheumatology (Oxford) 49: 231-239.

39. Apostolakos J, Durant TJ, Dwyer CR (2014) The enthesis: a review of the tendon-tobone insertion. Muscles Ligaments Tendons J 4: 333-342. [Crossref]

40. Sharma P, Mafffulli N (2006) Biology of tendon injury: healing, modeling and remodeling. J Musculoskelet Neuronal Interact 6: 181-190

41. Reinholt FP, Hultenby K, Heinegard D, Marks SC Jr, Norgard M, et al. (1999) Extensive clear zone and defective ruffled border formation in osteoclasts of osteopetrotic (ia/ia) rats: implication for secretory function. Exp Cell Res 15: 477-491.

42. Oliva F, Barisani D, Grasso A, Maffulli N (2011) Gene expression analysis in calcific tendinopathy of the rotator cuff. Eur Cell Mater 21: 548-557.

43. Globus RK, Doty SB, Lull JC, Holmuhamedov E, Humphries MJ, et al. (1998) Fibronectin is a survival factor for differentiated osteoblasts. J Cell Sci 111: 1385-1393.

44. Kamegai A, Tanabe TI, Masahiko Mori (1994) Laminin and fibronectin in bone induced by bone morphogenetic in mouse muscle tissue formation protein (BMP). $J$ Bone Miner Met 31: 125-135.

45. O’Brien EJ, Frank CB, Shrive NG, Hallgrìmsson B, Hart DA, et al. (2012) Heterotopic mineralization (ossification or calcification) in tendinopathy or following surgical tendon trauma. Int J Exp Pathol 93: 319-331.

46. Giai Via A, McCathy MB, Francke M, Oliva F, Mazzocca AD, et al. (2018) Hyperglicemia induces osteogenic differentiation of bone marrow derived stem cells: an in vitro study. Muscles Ligaments and Tendons Journal 8: 1-7.

47. Oliva F, Berardi AC, Misiti S, Maffulli N (2013) Thyroid hormones and tendon: current views and future perspectives. Concise review. Muscles Ligaments Tendons Journal 3: 201-203. [Crossref]

48. Berardi AC, Oliva F, Berardocco M, la Rovere M, Accorsi P, et al. (2014) Thyroid hormones increase collagen I and cartilage oligomeric matrix protein (COMP) expression in vitro human tenocytes. Muscles Ligaments Tendons Journal 4: 285-291.

49. Boivin GP, Elenes EY, Schultze AK, Chodavarapu H, Hunter SA, et al. (2014) Biomechanical properties and histology of $\mathrm{db} / \mathrm{db}$ diabetic mouse Achilles tendon. Muscles Ligaments Tendons Journal 4: 280-284.

50. Snedeker JG, Gautieri A (2014) The role of collagen crosslinks in ageing and diabete - the good, the bad, and the ugly. Muscles Ligaments Tendons J 4: 303-308. [Crossref]

Copyright: (C2020 Giai Via A. This is an open-access article distributed under the terms of the Creative Commons Attribution License, which permits unrestricted use, distribution, and reproduction in any medium, provided the original author and source are credited. 